\title{
A Survey on non-DTN and DTN Geographic based Protocols for VANETS
}

\author{
Radityo Anggoro ${ }^{a^{*}}$, Royyana Muslim I ${ }^{\mathrm{a}}$ and M. Hatem A A ${ }^{\mathrm{b}}$ \\ anstitut Teknologi Sepuluh Nopember, Surabaya, Indonesia. \\ 'Sana'a University, Sana'a, Yemen. \\ *Corresponding Author: onggo@ if.its.ac.id
}

\begin{abstract}
Vehicular Ad-hoc Network (VANET) is the extended version of Mobile Ad-hoc Network (MANET). Both share similar characteristics except VANET is utilizing the vehicles instead of mobiles. However; the high speed of the vehicles, the rapidly change of VANET topology, and the city environment characteristics, has become today's issues in terms of wireless communication using vehicles. In terms of routing protocols, there are so many protocols that could be used by VANETs. However; these protocols are not capable enough to achieve the communication. Position-based or geographical-based routing is one of the examples that we will cover in this paper. Our main focus is to make comparison of geographical-based routing protocols. For example, qualitative comparison of protocols that are used for packages' carrying and forwarding such as some Delay Tolerant Network (DTN) and non-DTN geographic routing protocols. Finally, each protocol has its own advantages and disadvantages. So it is important to have an idea about them in order to guidance the researcher. Depends on this comparison it is observed that hybrid DTN and non-DTN will perform better since it will take the advantages of all DTN and non-DTN.
\end{abstract}

Keywords: DTN and non-DTN geographical routing protocols, VANET.

\section{Introduction}

Recently vehicular networks has become very popular. The need for this kind of commination is significant since the development of communication technologies. Vehicular ad hoc networks are one of mobile ad hoc networks types that are used to make connection between vehicles. Nowadays, there are so many research interests in VANETs. In this type of mobility communication there are two types of entities: access points and vehicles those acts as the nodes ${ }^{(1)}$. VANETs are temporal networks which are self-organized by vehicles to route the packets. However, it is not easy to establish endto-end path between source and destination by utilizing only vehicle-to-vehicle $(\mathrm{V} 2 \mathrm{~V})$ communication, because the communication range of vehicle is limited and the movement of vehicle is very fast. Even if the communication between vehicles and infrastructures can be possible, network partition still exists where there is no infrastructure. Thus, most of the studies assume that the vehicles are always connected to the networks; thus they could not overcome the network partition problem In VANETs ${ }^{(2)}$, there are three ways of communications as shown in Figure 1:inter-vehicle communication, vehicle-to-roadside communication, and routing-based communication.

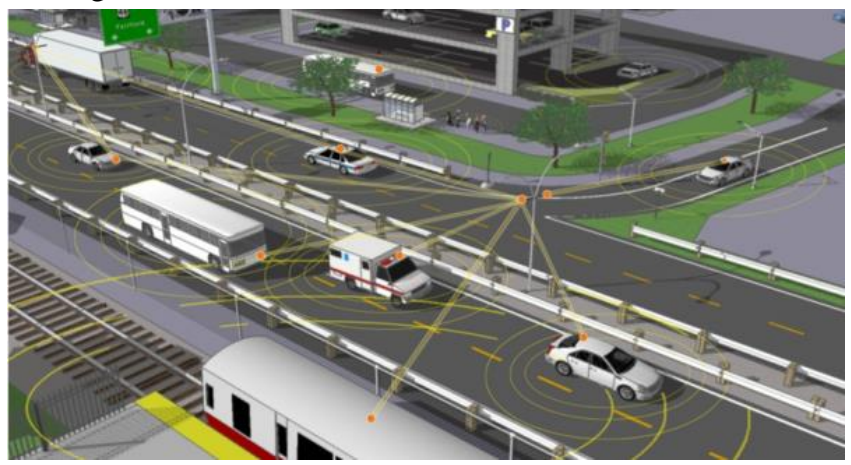

Fig.1. VANETs Communication (14).

In inter-vehicle communication, there are two types of message forwarding mechanism: naive broadcasting, where vehicles periodically broadcast messages and ignore the message from behind ${ }^{(3)}$. Our categorization for VANET communication is more general while we referred to the communication between the vehicle and the infrastructure 
networks as the infrastructural domain, where the infrastructure network could be any host such as the internet and other hosts for non-safety applications ${ }^{(4)}$. It ensures all the vehicles moving in forward direction can receive the broadcast message. Intelligent broadcasting where the number of message broadcast for an event is limited. If the event-discovery vehicles receive same messages from the vehicles from behind, it is assumed that at least one vehicle from behind received the message and stop broadcasting. The vehicle from behind is responsible for moving the message forward. Vehicle to roadside communication is the communication between the road-side unit and the vehicles. The roadside unit will periodically broadcast certain information such as speed limit to all the vehicles within its range. This communication must offer large bandwidth between the vehicle and roadside unit. Routing-based communication is a unicast communication from the source to the destination. The interesting aspect in VANET is that the node itself can act as a router or terminal.

Delay Tolerant Network (DTN) is a network communication that aim to connect and transfer the packets between the nodes even with the absent of the path between the source and the destination. In addition, this kind of communication defined an architecture based on a storecarry and forward paradigm ${ }^{(4)}$ that makes it very useful in terms of Ah-hoc Networks such as Vehicular Ad-hoc Networks. A de-lay tolerant network needs hardware that can store large amounts of data. Such media must be able to continue extended power loss and system restarts. It must also be available at any time. For this purpose include hard drives and high-volume flash memory. The data stored on these media must be organized and ranked by software that ensures accurate and reliable store-and-forward functionality ${ }^{(5)}$. In terms of VANETs DTN protocols are very useful to ease and assure the connection between the sender node and its destination even if there is a delay or far distance between them since the availability of the idea of store-carry and forwards. Using DTN protocols traffic can be as follows either packets are always transmitted, congregated and tested before any other data from that source to a given destination, packets have been successfully gathered at their proposed destination. Bulk traffic is not dealt with until all packets of other classes from the same source for the same destination should successfully transmitted and get back together again. There are two types of the concept of delay tolerant networks in vehicular ad-hoc networks namely, Delay Tolerant Networks (DTN) and non-DTN protocols which will be explained in details.
The importance of this paper is as follows. We believe that geographical routing protocols can help for managing roads condition by sharing the traffic information between vehicles that can helps the drivers to avoid the accidents. There are many survey papers that consider geographical routing protocols since the significant contribution that performed by these protocols such as they do not relay on the routing tables but they rely on the geographic position information that can be obtained from GPS, Depends on that, geographical-based routing protocols are more suitable for mobile networks and VANTEs since the source is always aware bout the targeted node location. Therefore, this information allow a message to be routed to the destination without knowledge of the network topology or a prior route discovery However, as far as we know there is no paper focused on both DTN and non-DTN and even though there is. However, their focus is still different from this paper.

Our contribution is that, we are focusing on geographical based routing protocols and discuss both DTN and non-DTN routing strategies, strengths and limitations. However, we will focus on some well-known routing protocols from each category such as follows. In non-DTN Geographical-based protocols we discuss GSR and A-STAR. Moreover, the discussion will cover some popular DTN VANET Geographical-based protocols such as GeOpps (Geographical Opportunistic Routing), and Vehicular Assisted data delivery (VADD). This is because both abovementioned protocols not only used for packages' carrying and forwarding but also, all of them are popular in VANETs. Finally, by highlighting the problems of each under discussion protocol we end this survey with giving an opinion about the preferred protocol among these Geographical-based routing protocols which will be compared and proposed future direction.

\section{VANETs Geographic Routing Protocols}

VANET routing protocols can be basically divided into two main categories as follows. First, topology-based which means the node should be aware of the network to be able to forward packets relying on that information about the other nodes. Second, Position-based routing protocols this make a use of the Geographical Position System (GPS) and every node has enough information about the position of the other node ${ }^{(1)}$. However, our main focus is on the geographicalbased protocols which is a routing principle that relies on geographic position information that is provided by GPS. 
This kind of protocols is mainly proposed for wireless networks and depends on the idea that the source sends a message to the geographic location of the destination instead of using the network IP address. The idea of using position information to route and communicate with each other ${ }^{(1)}$. Geographic routing requires that each node can determine its own location and that the source will be able to be aware of the location of the destination. With this information a message can be routed to the destination without knowledge of the network topology or a prior route discovery.

There are two main classes of Geographic routing protocols: Delay Tolerant Network (DTN) Protocols, non-DTN Protocols $^{(3)}$ which we will explain in details in the next section.

\section{1 non-DTN Protocols}

As known geographical-based routing is the concept of forwarding the packets to the destination with having an idea about the destination position. However; this kind of VANET protocols consider the transmission of the packets as soon as possible and pay no attention to the interruption between nodes ${ }^{(5)}$. Therefore, forwarding can happen by submitting the packets to neighbor nodes or to the nodes those are closer to the destination. However when there is no node closer to the destination $^{(1)}$ the forwarding can be failed. So there are so many protocols that recover this failure. Each protocol has its own strategies and manners to recover this failure.it our pleasure to give a brief review about some non-DTN routing.

\section{(a) GSR (Geographic Source Routing)}

As in ${ }^{(5,6)}$ the main idea in GSR is the need of the global knowledge of the place (the map of a certain aimed place). It uses the Reactive Location Service RLS to locate the destination. This protocol merges the topological information and the position based routing. The sender determines the junction that has to be traversed by the packet using the Dijkstra's shortest path algorithm. Therefore, Forwarding between junctions is done in a position-based fashion and calculate the shortest distance between the sender and the reciever. It is designed for city environments where there are vehicles. Junctions are always placed on placeses that where the actual routing decisions are taken. Therefore, packets should always be forwarded to something called coordinator. This protocol is scalable for greedy forwarding.so the packets delivery is very high. The demanding node floods the network with a "location request" for a particular node identifier. When the node that corresponds to the requested identifier receives the location request, it sends a "location reply" back to the demanding node. However, the backwards of it can be as follows ${ }^{(7)}$ : this protocol neglects the situation like sparse network where there are not enough nodes for forwarding the packet. It also suffers from a high routing overhead because the use of the hello beacons as a control message in this protocol.

\section{(b) A-STAR}

A-STAR (Anchor-based Street and Traffic Aware Routing) ${ }^{(7)}$ is geographical based routing protocol. It is aimed to be used in a city environment. This protocol has many characteristics those are available in GSR. Moreover, this protocol depends on GSR to perform the routing task. However, this protocol includes the traffic density information to weight the streets of the scenario. In addition, A-STAR uses the street maps to compute the sequence of junctions (anchors) through which a packet must pass to reach its destination. The main difference between A-STAR and GSR is that A-STAR computes the anchor paths with traffic awareness. It is possible by using statistically rated maps such as (counting the number of the city bus on each street). With this improvement, these protocols can achieve higher connectivity among the nodes because of its trying to find an end-to-end connection. On the other hand, this protocol uses static information to find a path between the source and the destination; the packets delivery ratio is also low if compared with GSR. The main drawback of this protocol is that it becomes time consuming.

\subsection{DTN Protocols}

This type of geographical-based routing protocols consider computer network construction that try to address the technical issues in mixed networks that may lack of continuous connectivity, which effects in a lack of instantaneous end-to-end paths. This kind of protocols uses the concept of store-carry-and-forward strategies to overcome frequent disconnection of the network, great scales, long delays, limited bandwidth, power constraints and high faults rates ${ }^{(1)}$. DTN support that each node helps the other in forwarding and exchanging the packets between each other. The delay can occur because of the limited transmission range for transferring the packets. However DTN cannot guarantee the disconnection $100 \%$. There are some mechanisms that are used to maximize the probabilities of the packet the have been transferred to replicate many copies of the packet in hopes to guarantee the message arrival 
to the destination. There are so many DTN routing protocols that can handle the routes efficiently. Therefore, in this section we review some of these DTN protocols.

\section{(a) GeOpps: Geographical Opportunistic Routing}

As in ${ }^{(8)}$ This protocol consider all nodes recognize their own locations and their neighbor node geographic locations through position-pointing devices such as GPS. It applies a delay tolerant technique that the vehicles store the packets till the suitable condition for it to be forwarded. Therefore, it is considered as a DTN routing protocol. It is a forwarding protocol which exploits both the opportunistic nature of. The operation to select the next node can be as follows: neighbor vehicle that follows suggested routes to their drivers' destination calculate the nearest point that they will get to the destination of the packet; after that, they make a use of their maps and the nearest node to minimize the time needed for them to reach to the destination; The vehicle that can deliver the packet quicker/closer to its destination becomes the next packet carrier. In addition, a geographical delay tolerant routing algorithm that exploits information from the vehicles' map reading system to route messages to a particular location. It calculates the straight distance from the destination of packets to the nearest point (NP) of the vehicles' path and estimates the arrival time of a packet at the target node. It has a high delivery ratio that relies on the mobility patterns. This protocol is for delay tolerant data but its performance rely on exact the trajectory information. Therefore, if the node did not follow the GPS path the routing decision may be will be mistaken.

\section{(b) Vehicle-assisted data delivery (VADD)}

As it said in ${ }^{(9)}$ VADD is a vehicular geographical based on the idea of carry and forward routing protocols that their scheme intended to improve the routing process when there is disconnected vehicular networks based on the carryand-forward conception depending on the use of conventional vehicle mobility. Therefore, the most important issue is to select a forwarding path with the smallest packetdelivery delay. The node creates a decision at an intersection and selects the next forwarding path with the insignificant packet delivery delay. The path is only a split road from ${ }^{(9)}$ an intersection. The best path of the packet forwarding is selected by exchanging between three packet modes (intersection, straight way, and destination). They take the advantage of the GPS information since they are in need for the location maps to determine their targeted nodes. Their main goal is to select the path with the smallest packet delivery delay. The behavior of the protocol depends on the location of the node holding the message. The main backward of these kind of protocols that since the change of the topology and the traffic so, the delay will be very large.

\section{Non-DTN vs DTN Protocols}

In this section we would like to make a comparison between non-DTN routing protocols in some characteristics and challenges and also, the DTN protocols in some characters and challenges separately such as follows.

\section{1 non-DTN geographical Routing Protocols}

non-DTN routing Protocols can be compared in so many aspects. However; we will eliminated this comparison to the common characteristics as shown in Table 1 below since some of these protocols are aimed at providing vehicle-to vehicle communication while the others focus on vehicles to roadside communications. We all know the compared protocols in this section are position-based non-DTN protocols. Therefore they all make a use of GPS information to determine the destination of the packets. They all use greedy forwarding to transferring the packets to the nearest destination's neighbor. In some non-DTN protocols the node will hold the packet until discovering that destination's closest neighbor after that it will forward it.

Table 1. Comparison for non-DTN Protocols.

\begin{tabular}{|l|c|c|}
\hline & GSR & A-STAR \\
\hline Vehicle-vehicle & Yes & - \\
\hline Vehicle-infrastructure & - & - \\
\hline Connection oriented & Yes & Yes \\
\hline Buffering & & \\
\hline The need of the Map & Yes & Yes \\
\hline Greedy forwarding & Yes & Yes \\
\hline Speed & - & Medium \\
\hline Latency & Medium & - \\
\hline Suitable Environment & Town & Town \\
\hline
\end{tabular}

\subsection{DTN geographical Routing Protocols}

In this part we will compare some DTN geographicalbased routing protocols which are commonly used for VANETs. Table 2 will show the main compared characteristics. All of these compared DTN protocols are position-base since they make a use of GPS information to determine the position of their destinations. In addition all of them depend on the digital map as well. 
Table 2. Comparison for DTN Protocols.

\begin{tabular}{|l|c|c|}
\hline & VADD & GeoOpps \\
\hline Buffering & Yes & Yes \\
\hline Map needed & Yes & Yes \\
\hline Delay/tolerant & Yes & Yes \\
\hline Greedy needed & - & - \\
\hline Single copy & Yes & Yes \\
\hline multiple copy & - & - \\
\hline Time to live & - & - \\
\hline Traffic aware & Yes & - \\
\hline Suitable Environment & Town & Town \\
\hline
\end{tabular}

\subsection{Discussion}

To illustrate the capability of the selected and voted protocols and their unique characteristics and mechanisms we have discussed some of these characteristics and give the reasons why we have chosen them. This is because we target the other reader to know the exact difference between those mentioned protocols as follows. For example; Geographic routing depend on essentially on position information and other mobility parameters provided by geographic placing devices such as global positioning systems (GPSs). This means the protocols is in need for the map that can help it to determine the distance between the source and distention. Furthermore, the need for the map can help that protocol to locate the position of the destination.

In terms of buffering (store carry and forward) this feature is the main different which have given to the geographical protocols and made them differ from the topology protocols those relay on e.g routing table to forward and exchange their packets.

The greedy forwarding feature helps the nodes to determine their destinations with high possibility that helps the node the exact position of the other node that will receive its packet it could be the destination or another node that is closer to the destination. In addition, most of the single path strategies depends on greedy forwarding that helps the source node to determine to it can forward its packet.

Delay Tolerant feature is the key point that differ the DTN from non-DTN routing protocols since DTN more reliable about the arrival of the packet to the destination without paying any attention about the latency that will happen in the network. This kind of protocols is preferred to work in the hometowns of highways-where the availability of the nodes is small-since the carrier of the packet will keep that packet till it finds the exact receiver for it. However, non-DTN routing protocols do not guarantee the arrival of the packets to the destination since their destination but they pay more attention on how to forward the packet within a small period of time. Therefore, this kind of protocol will be useful in the urban environments since there is the possibility of the packets arrival could be reasonable.

Traffic aware matrix is to determine the exact traffic of the nodes. This traffic aware matrix makes use of the location-maps to help it to determine that the density of the nodes is high or low.

Finally in terms of time to live this feature has make the protocol unique on solving the overload on the networks by giving the packet a limited time to stay carrier on the node till it can forwarded to the other. Moreover, this feature helps for minimizing the number of the buffered packets on the network.

\section{Conclusion}

This paper has surveyed the Geographical-based routing protocols which can be classified into two popular types, DTN and non-DTN so we have compared four protocols in each DTN and non-DTN geographical-based routing protocols in terms of their mechanisms, performance, buffering, and so on. We can conclude that in each protocol has its own advantages and backwards. Therefore, the best network is the one which is able to fulfill its requirement. For example, some network needs the faster transmission even though, with packet lost so we can use non- DTN geographical-based routing protocols. On the other hand, some networks consider the validation of the data even though there is a delay so we can use DTN Geographicalbased routing protocols.

\section{Future Work}

A large number of Geographical-based routing protocols either DTN or non-DTN have been recently proposed by so many authors who have their own different ideas so these protocols are still holding advantages and weaknesses under different conditions. However, our efforts are mainly to fulfill the details of the essential Geographical-based routing protocols. In this paper, we focused completely on mechanisms for addressing such as the case where a connected path is not available from source to destination and so on. We expect that a hybrid approach that first attempts to use end-to-end Geographical-based routing 
protocols and falls back to the maintained Geographicalbased routing protocols above. Further, it may be possible to exploit the advantages and the ideas of the others and propose such hybrid techniques as future protocols that can improve the transmission performance. We need to consider the condition of DTN protocols that assure the packet arrival to its target even if, there is a delay and the condition of Non DTN protocols that consider the use of the storage and the speed while transmitting the data without considering the packet arrival. Out of that, we need to propose a Hybrid protocol that combine and take the advantages of these conditions out of both DTN and non-DTN as possible as it can.

\section{References}

(1) Kumar, Aashish, and Mack Tyagi, (2014), "Geographical Topologies of Routing Protocols in Vehicular Ad hoc Networks-A Survey." International Journal of Computer Science \& Information Technologies vol. 5, no. 3, pp. 3062-3065.

(2) Nabil Benamar, Kamal D. Singh, Maria Benamar, Driss El Ouadghiri, Jean-Marie Bonnin.(2014), " Routing protocols in Vehicular Delay Tolerant Networks A comprehensive survey." Computer Communications 48 , pp. 141-158.

(3) Sardar Muhammad Bilal, Carlos Jesus Bernardos,Carmen Guerrero,(2013) "Position-based routing in vehicular networks: A survey," J. Netw. Comput. Appl., vol. 36, no. 2, pp. 685-697.

(4) Saif Al-Sultan, Moath M. Al-Doori, Ali H. AlBayatti,Hussien Zedan,(2014) "A comprehensive survey on vehicular Ad Hoc network," J. Netw. Comput. Appl., vol. 37, no. 1, pp. 380-392.

(5) BaraaT.Sharef a, RaedA.Alsaqour a,n, MahamodIsmail,(2014) "Vehicular communication ad hoc routing protocols: A survey," J. Netw. Comput. Appl., vol. 40, no. 1, pp. 363-396.

(6) B. Paul, M. Ibrahim, and M. Abu Naser Bikas, "VANET Routing Protocols: Pros and Cons," Int. J. Comput. Appl., vol. 20, no. 3, pp. 28-34, 2011.

(7) Boon-Chong Seet, Genping Liu, Bu-Sung Lee, ChuanHeng Foh, and Kai-Juan Wong, (2014) “A-STAR: A mobile ad hoc routing strategy for metropolis vehicular communications," Third Int. IFIP-TC6 Netw. Conf. Athens, Greece, May 9-14, 2004, Proc., vol. 3042.
(8) Ilias Leontiadis and Cecilia Mascolo, (2007)“GeOpps: Geographical opportunistic routing for vehicular networks," 2007 IEEE Int. Symp. a World Wireless, Mob. Multimed. Networks, WOWMOM.

(9) Jing Zhao, Student Member, IEEE, and Guohong Cao, Senior Member, IEEE,(2008,May) "VADD : VehicleAssisted Data Delivery in," IEEE Transactions On Vehicular Technology, Vol. 57, No. 3, pp. 1910-1922. 